\title{
Two-types of Hydroxyapatite Coating Implant : A Retrospective Clinical Study
}

Kim Sang Yun ${ }^{1 *}$, Kim Young-Kyun ${ }^{1,2}$

${ }^{1}$ Department of Oral and Maxillofacial Surgery, Section of Dentistry, Seoul National University Bundang Hospital, Seongnam , Korea

${ }^{2}$ Department of Dentistry \& Dental Research Institute, School of Dentistry, Seoul National University, Seoul, Korea

\section{Introduction}

Clinical research has shown that hydroxyapatite coated implants can improve the outcome of this procedure by enhancing the osseointegration process, decreasing healing time, and increasing the 5-year success rates post procedure.

\section{Purpose}

We conducted a retrospective descriptive study to evaluate the clinical outcomes of two hydroxyapatite (HA) coated implants, OT (Osstem TS III-HA, Osstem implant Co., Busan, Korea) and ZM (Zimmer TSV-HA, Zimmer dental, Carlsbad, USA.

\section{Materials and Methods}

Subjexts: 303 implants (89 Osstem TS III-HA, 214 Zimmer TSV-HA), which were placed in the section of dentistry, Seoul National University Bundang Hospital from 2010 to 2012
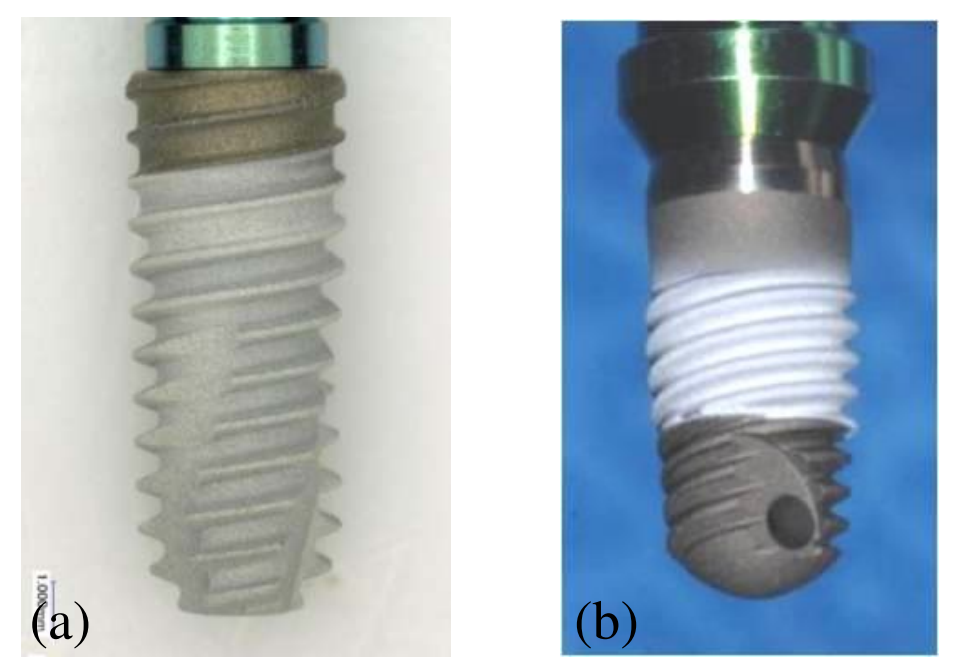

(a) Osstem TS III-HA

(b) Zimmer TSV-HA

Table 1. Classification by Surgical procedure

\begin{tabular}{|cccc|}
\hline & Osstem & Zimmer & Total \\
\hline 1-stage surgery & 63 & 137 & 200 \\
2-stage surgery & 26 & 77 & 103 \\
Total & 89 & 214 & 303
\end{tabular}

Table 2. Classification by prosthetic loading time

\begin{tabular}{|cccc|}
\hline & Osstem & Zimmer & Total \\
\hline Immediate Loading & 34 & 34 & 68 \\
Early Loading & 7 & 18 & 25 \\
Conventional Loading & 31 & 112 & 143 \\
Delayed Loading & 17 & 50 & 67 \\
Total & 89 & 214 & 303 \\
\hline
\end{tabular}

\section{Prognosis criteria}

1) Annual marginal bone loss

: Periapical taking (crestal bone loss)

2) Primary stability

3) Secondary stability

: Osstell Mentor (ISQ)

4) Success rate

: Albrektsson(1994)

(1) not more than $1.0 \mathrm{~mm}$ of marginal bone loss during first year of loading

(2) not more than $0.2 \mathrm{~mm}$ resorption per year

5) Survival rate

6) Prosthetic complications

\section{Results}

1) Overall, there were no significant differences between $O T$ and ZM when comparing success rates, survival rates, and annual marginal bone loss; however, there was a difference in late stability (ISQ). Specifically, ISQ, which occurs after healing, was slightly higher in the in the OT implants $(77.83 \pm 8.23$ vs. $76.09 \pm 6.90, \mathrm{P}<.05)$.

Table 3. Total average results

\begin{tabular}{|cccc|}
\hline Total average & Osstem & Zimmer & $\begin{array}{c}\text { P value } \\
(\boldsymbol{\alpha}=\mathbf{0 . 0 5})\end{array}$ \\
\hline $\begin{array}{c}\text { Annual Bone Loss } \\
(\mathbf{m m} / \mathbf{y r})\end{array}$ & $-0.27 \pm 0.89$ & $-0.32 \pm 0.70$ & 0.059 \\
$\mathbf{1}^{\text {st }} \mathbf{I S Q}$ & $69.20 \pm 15.00$ & $68.45 \pm 11.26$ & 0.152 \\
$\mathbf{2}^{\text {nd }} \mathbf{I S Q}$ & $77.83 \pm 8.23$ & $76.09 \pm 6.90$ & 0.030 \\
Success rate (\%) & 92.13 & 94.44 & 0.821 \\
Survival rate (\%) & 97.75 & 98.5 & 0.449 \\
\hline
\end{tabular}

2) When analyzing the healing periods between the two implant types, only immediate loading showed a statistically significant difference $(\mathrm{P}<.05)$. The OT implants performed better than the ZM implants when comparing annual marginal bone loss $(-0.17 \pm 0.58 \mathrm{~mm} / \mathrm{year}$ vs $0.45 \pm 0.80 \mathrm{~mm} / \mathrm{year})$ and late stability $(84.36 \pm 3.80$ ISQ vs 82.48 \pm 3.69 ISQ, $\mathrm{P}<.05)$.

Table 4. Classification by immediate loading

\begin{tabular}{|cccc|}
$\begin{array}{c}\text { Immediate } \\
\text { loading }\end{array}$ & Osstem & Zimmer & $\begin{array}{c}\text { P value } \\
(\boldsymbol{\alpha}=\mathbf{0 . 0 5})\end{array}$ \\
\hline $\begin{array}{c}\text { Annual Bone Loss } \\
(\mathbf{m m} / \mathbf{y r})\end{array}$ & $-0.17 \pm 0.58$ & $-0.45 \pm 0.80$ & 0.005 \\
$\mathbf{2}^{\text {nd }} \mathbf{I S Q}$ & $84.36 \pm 3.80$ & $82.48 \pm 3.69$ & 0.007
\end{tabular}

Table 5. Classification by 1-stage surgery

\begin{tabular}{|cccc|}
\hline 1-stage & Osstem & Zimmer & $\begin{array}{c}\text { P value } \\
(\boldsymbol{\alpha}=\mathbf{0 . 0 5})\end{array}$ \\
\hline $\begin{array}{c}\text { Annual Bone Loss } \\
(\mathbf{m m} / \mathbf{y r})\end{array}$ & $-0.23 \pm 0.55$ & $-0.3 \pm 0.54$ & 0.010 \\
$\mathbf{2}^{\text {nd }}$ ISQ & $80.95 \pm 6.18$ & $\begin{array}{c}77.73 \pm 6.3 \\
0\end{array}$ & 0.001 \\
\hline
\end{tabular}

3) There were no statistically significant differences in the remaining indices, which were early, conventional, and delayed loading ( $\mathrm{P}>.05)$. Finally, OT appeared to have slightly better bone loss and late stability than ZM in 1stage surgery.

\section{Conclusions}

Both OT and ZM implants had favorable survival rates $(97.75$ vs $98.5 \%$, respectively). Although OT performed slightly better than $\mathrm{ZM}$ in the immediate loading and 1-stage surgery, there were not many statistically significant differences between the two implant types when comparing most of the indices. 